\title{
Bioactive Interlinked Extracellular Matrix-Like Silicon Nano-Network Fabricated by Femtosecond Laser Synthesis
}

\author{
Priyatha Premnath, Bo Tan, ${ }^{1}$ and Krishnan Venkatakrishnan ${ }^{1}$
}

\begin{abstract}
Nanostructured silicon has proven to be a promising candidate in tissue engineering. However, recent research on fabrication of silicon scaffolds has been limited to expensive, complex, and time-consuming lithographic techniques that require the addition of caustic chemicals. Moreover, these techniques generate structures that do not truly mimic the extracellular matrix $(\mathrm{ECM})$. Therefore, we introduce a novel, interlinked, silicon nanonetwork fabricated by $\mathrm{MHz}$ ultrafast laser synthesis. We demonstrate that ultrafast laser synthesis is simple, rapid, free of any chemical additions, and can be carried out under ambient conditions. Variation in laser parameters resulted in an alteration in the pore size and density of the silicon fibrous network. Microscopic analysis revealed a highly charged silicon network with elevated adhesion forces. In vitro bioactivity tests indicate the precipitation of bone-like apatite in just 3 days. Cell proliferation studies on the silicon nano-network present a 300\% increase in comparison to its bulk counterpart. Scanning electron microscopy analysis shows healthy migration and attachment of cells on the silicon nano-network. This study points to a correlation between elevated cell proliferation and the ECM-like structure of the silicon nano-network. This ECM-like silicon nano-network suggests significant potential not only in tissue engineering and regeneration but also in other biomedical applications such as biosensor detection.
\end{abstract}

Key words: biomaterials; extracellular matrix; tissue engineering

\section{Introduction}

$\mathbf{T}$ HE SYNERGY OF materials engineering and cell biology has resulted in the development of novel biomaterials that could perform as a tissue scaffold. A tissue scaffold is required to provide a three-dimensional (3D) environment for cells to grow and ultimately form a tissue. An ideal scaffold would mimic the extracellular matrix (ECM) that is created by the cells of each tissue in the body. ${ }^{1}$ In detail, an ideal scaffold should possess the characteristics of bioactivity, three dimensionality, promotion of cell adhesion, and biocompatibility. ${ }^{2}$ In addition, cells in their natural environment are surrounded by nanoscale pores, fibers, and crystals. Nanostructured metals, polymers, alloys, and ceramics have all been well researched as potential scaffold materials. ${ }^{3-7}$ Nanostructured silicon, owing to its biocompatible and biodegradable nature, has attracted much recent attention. However, unlike other materials mentioned earlier, only very limited research work has been carried out with the aim of investigating the potential of nanostructured silicon as a scaffold material. This may be attributed to the fact that earlier nanostructured silicon demonstrated relatively low bioactiv- ity compared with other nanostructured materials. Bioactivity is a vital characteristic for an ideal scaffold. Bioactive materials form a bond between the tissue and the implant material or osseointegration, which includes the nucleation of hydroxyapatite. If the bonding interface is not established, then a fibrous tissue is formed around the implant, causing fibro-osseous integration. This results in failure of the scaffold and rejection from the surrounding tissues. There has been some work done to study bioactivity on silicon. For instance, mesoporous silicon showed precipitation of calcium phosphate after a period of two weeks, whereas a glass-ceramic scaffold showed precipitation in less than one week. ${ }^{8,9}$ Bioactive glass with high silicon content was studied as an alternative to overcome this drawback. However, bioactive glasses are very expensive.

Thus far, silicon micro/nanoparticles, porous silicon micro/nanostructures, and silicon nanowires have been investigated for the purpose of a tissue scaffold. Coffer et al. showed the precipitation of calcium phosphate on mesoporous silicon. ${ }^{8}$ This early work proves that even though bulk silicon is bio-inert, mesoporous silicon demonstrates bioactivity. Further, Canham showed that it took 7 days to see

Departments of ${ }^{1}$ Mechanical and Industrial Engineering and ${ }^{2}$ Aerospace Engineering, Ryerson University, Toronto, Canada. 
bioactivity in microporous silicon. ${ }^{10}$ With nanostructures, it is expected to see increased bioactivity, as nano-scaling would enable higher bioactivity. Bioactive substrates should also promote cell growth and proliferation. Recent studies have shown cell proliferation on different silicon micro/nano/ meso structures. A case in point, Sun et al. showed that cell proliferation increases on silicon substrates with micropores when compared with meso- and nanopores. ${ }^{11}$ Agrawal et al. deposited a nanoporous silicon thin film of $55 \mathrm{~nm}$ thickness on a silicon wafer, which induced significantly higher cell proliferation than the conventional cell growth substrates and monolayer membranes to study cell-cell communication. ${ }^{12}$ The fabrication of silicon protrusions was studied as a possible scaffolding candidate by Ranella et al., where micrometer spikes were created with nano protrusions. ${ }^{13} \mathrm{How}$ ever, research has shown that such protrusions penetrate the cells and prevent them from forming focal adhesions. ${ }^{14}$ Sputter-coating microstructures of metals with siliconsubstituted hydroxyapatite containing varying quantities of silicon were researched for the purpose of fabricating a scaffold. ${ }^{15}$ Here, increasing the content of silicon showed better cell proliferation with mature cytoskeletons. This research cemented the fact that silicon is, in fact, a viable scaffolding material. Another study done by Kim et al. demonstrated that silicon nanowires of a diameter of $90 \mathrm{~nm}$ and a length of $6 \mu \mathrm{m}$, which were grown on a silicon substrate, were able to sustain cells for several days. ${ }^{16}$ In all these works, silicon is fabricated into individual one-dimensional or two-dimensional nanostructures that are assembled in certain patterns. Even though none of them resemble the ECM in terms of structure and morphology, interlinking and random assembly of nanostructures, these silicon nanostructures demonstrated a marked increase in cell growth and proliferation. ${ }^{17-19}$ To the best of our knowledge, no studies have developed or examined the effect of ECM-like nanostructures of silicon on cell growth/proliferation and bioactivity.

Our group's recent research has demonstrated a novel method through which silicon and many other materials can form unique nano-networks through $\mathrm{MHz}$ frequency ultrafast laser synthesis. ${ }^{20}$ This fabrication is done in ambient atmospheric conditions and does not need the addition of any harmful material (i.e., catalyst). The interlinked nanofibrous structure of the nano-network makes them particularly suitable for cell attachment. Figure 1 depicts the complete fabrication and experimental study conducted on this silicon nano-network. This article describes the fabrication and characterization of silicon nano-networks. Cell-material interaction and bioactivity of these nano-networks is also reported.

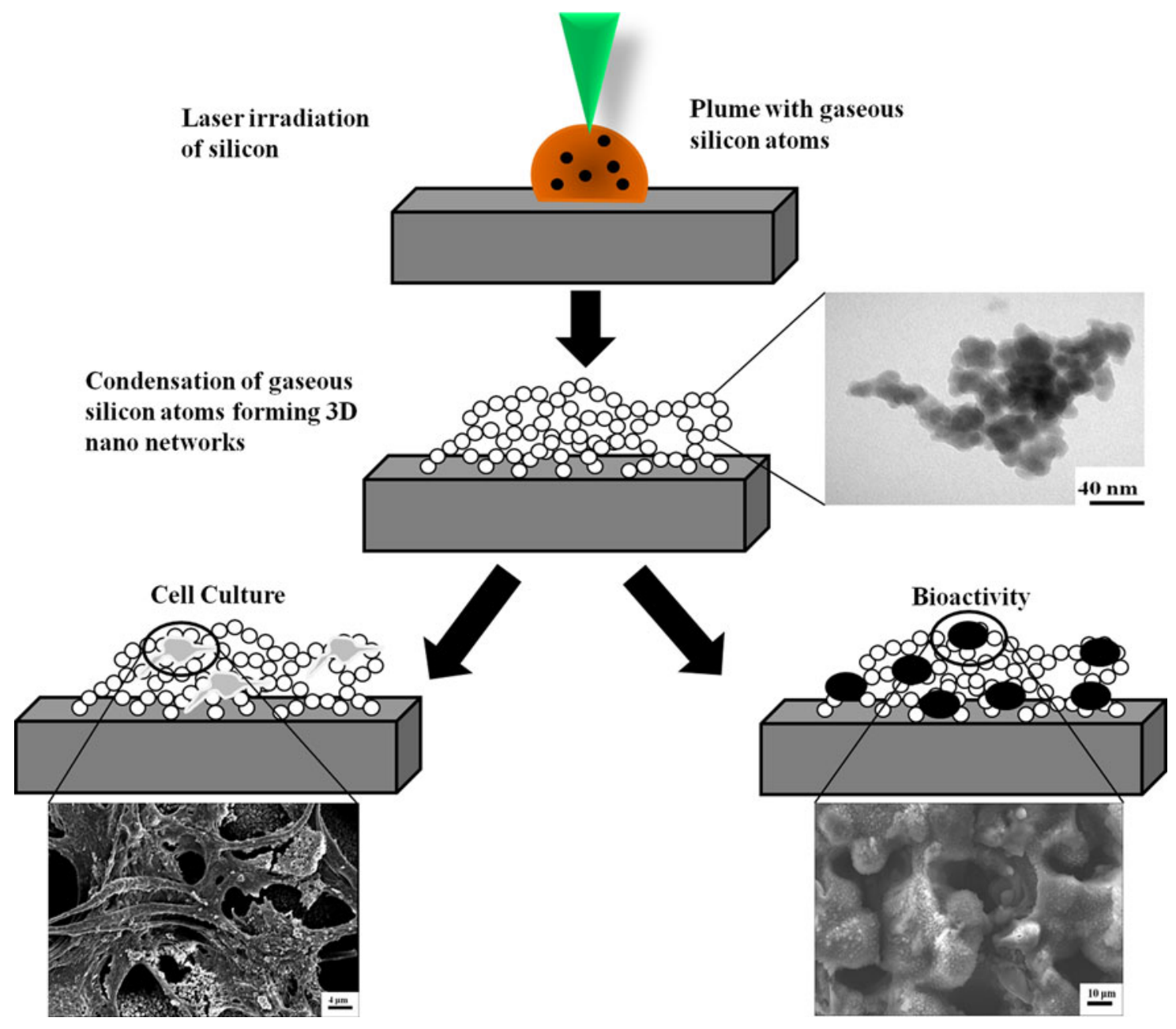

FIG. 1. Graphical illustration of development and testing of a silicon nano-network. 


\section{Materials and Methods}

All materials were purchased from Sigma Aldrich, unless specified otherwise.

An undoped $<100>$ oriented silicon wafer (University Wafers) with $500 \mu \mathrm{m}$ thickness was cut into $4-\mathrm{cm}^{2}$ squares. The squares were washed with deionized water and then dried. These substrates were irradiated using a diodepumped, Yb-doped femtosecond laser system (Clark-MXR, Inc.; IM-PULSE Series ultrashort pulse laser) at $26 \mathrm{MHz}$ with laser material interaction times (dwell time) between 1 and $15 \mathrm{msec}$ at a constant power of $15 \mathrm{~W}$.

The obtained silicon nano-network was characterized with a scanning electron microscope (SEM) (Hitachi S 5200) and a transmission electron microscope (TEM) (Hitachi H 700 CTEM). Analysis of pore size and particle size was carried out using ImageJ (available from the NIH website).

Measurement of zeta potentials of the nano-networks was carried out using a zetasizer (Nano-ZS90; Malvern Instruments). The silicon nano-networks were mixed in $1 \mathrm{~mL}$ of deionized water and transferred to a cuvette, after which measurements were taken.

Force of adhesion of the nano-networks was measured using an atomic-force microscope (AFM; NTEGRA, NTMDT). A single crystal silicon tip (antimony doped) with a spring constant of $0.11 \mathrm{~N} / \mathrm{m}$ was used in contact mode. NTMDT software was used to calculate the force of adhesion from the obtained graphs.

To test bioactivity, simulated body fluid (SBF) was made following the procedure detailed by Kokubo and Takadama and maintained at $4^{\circ} \mathrm{C} .^{21}$ A calculated amount of SBF was heated to $36.5^{\circ} \mathrm{C}$ in a container into which the samples with nanonetworks were deposited. To prevent gravitational effects, samples were placed perpendicular to the base of the container.
The soaked samples were allowed to incubate for 3 and 10 days. The samples were then removed, dried, and analyzed using SEM and energy-dispersive X-ray spectroscopy (EDX).

Cell-material interaction was tested with NIH 3T3 mouse embryonic fibroblasts (ATCC). Silicon substrates with nanonetworks and control samples (plain silicon) were attached to 96-well plates using bioinert glue (Edmund Optics). The cells were grown to confluence in tissue culture flasks and then seeded onto silicon substrates attached to 96-well plates at a density of $10^{5}$ cells $/ \mathrm{mL}$. The seeded silicon substrates were incubated for $48 \mathrm{~h}$. MTT assay was carried out to measure cell proliferation. SEM was performed to assess the attachment morphology of cells. After $48 \mathrm{~h}$ of incubation, MTT assay and SEM analysis were performed to measure proliferation and attachment, respectively.

For the MTT assay, media were aspirated from the wells, and $2.5 \mathrm{mg} / \mathrm{mL}$ of MTT (3-(4,5-dimethylthiazol-2-yl)-2,5-diphenyltetrazolium bromide, a yellow tetrazole) prepared in Dulbecco's phosphate-buffered saline was added to each well. After another $4 \mathrm{~h}$ of incubation, dimethyl sulfoxide was added to dissolve the insoluble formazan crystal. ${ }^{22}$ Absorbance was measured using a UV-Visible spectrophotometer (Shimadzu) at $540 \mathrm{~nm}$.

For SEM analysis, the cells were fixed using $2 \%$ glutaraldehyde and dehydrated in increasing concentrations of ethanol. After drying, the images of samples at room temperature were taken at varying magnifications using an SEM (Hitachi SU1510).

\section{Results and Discussion}

\section{Characterization of silicon nano-network using SEM and TEM}

Figure 2a shows an SEM image of the silicon nanonetwork. The mechanism of nano-network formation is clearly
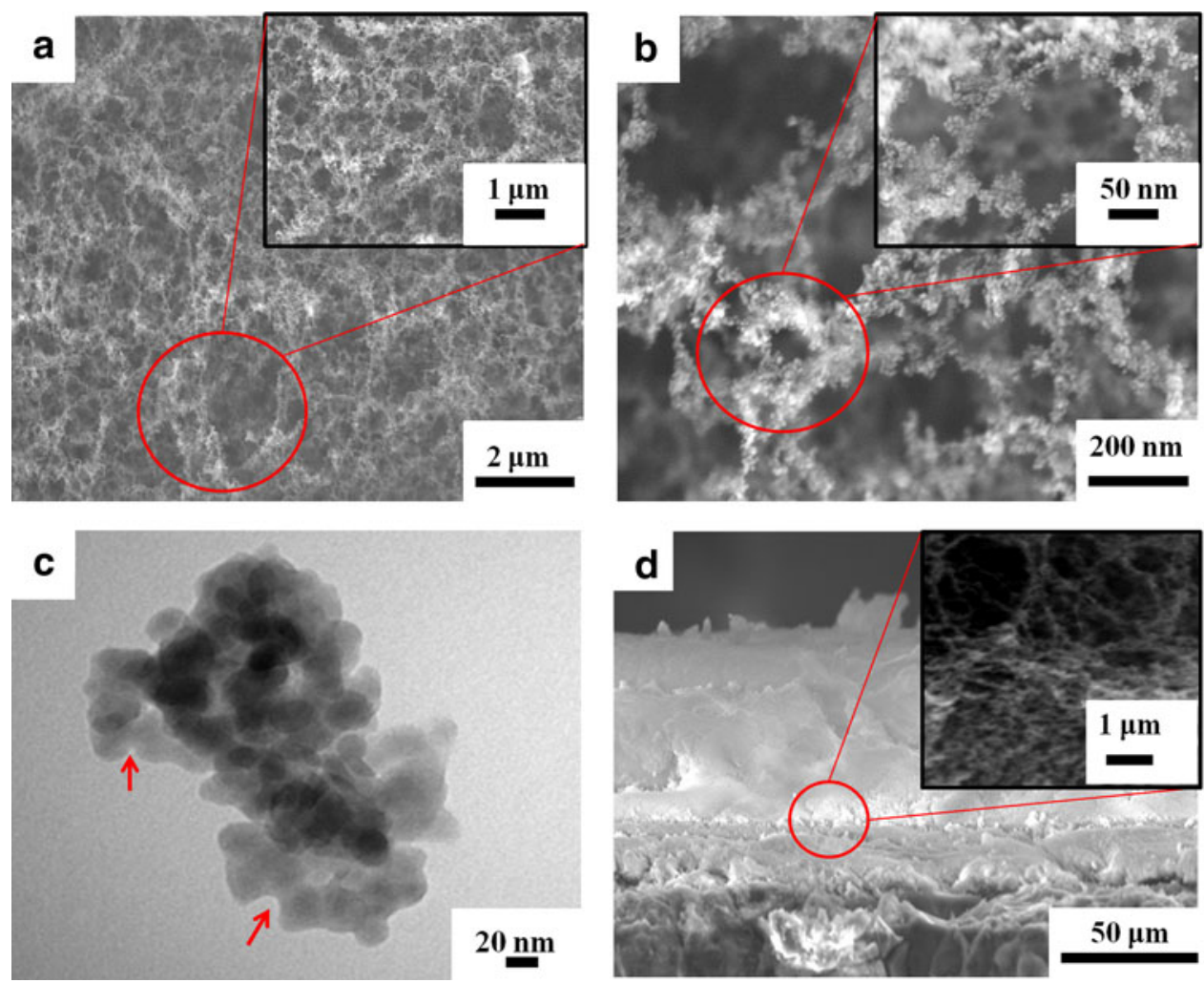

FIG. 2. (a, b) SEM images of a silicon nano-network. (c) TEM of silicon nanoparticles that combine to become a silicon nano-network. (d) Cross-sectional SEM of a silicon nano-network showing decreasing density with an increase in height. SEM, scanning electron microscope; TEM, transmission electron microscope. 


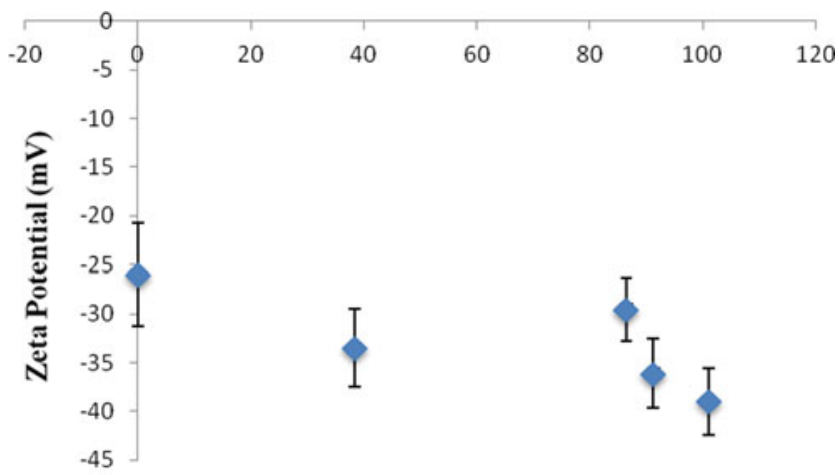

Thickness of fibers $(\mu \mathrm{m})$

FIG. 3. Zeta potentials versus deposition thickness, with $0 \mu \mathrm{m}$ thickness being the control sample.

explained in Tan et al. ${ }^{20}$ Aggregation of the individual nanoparticles is more fusion than mere loose packing, and, hence, the bonds between the molecules are very strong (Fig. 2b). ${ }^{23,24}$ This aggregation leads to a fibrous $3 \mathrm{D}$ nanostructure that is interlinked between layers. The surface charge of these nanoparticles is considerably high (Fig. 2c). Figure 2c also illustrates the formation of necks caused by fusion.

The SEM image depicting the nano-network shows that silicon nanoparticles aggregate to form chains, rings, and bridges. These structures are interconnected, 3D, and porous.
Furthermore, these structures are assembled randomly and display no particular order or pattern. The thickness, pore size, and particle size of this nano-network can be varied using different laser parameters. A cross-section of the nano-network shows that at the substrate-nano-network interface (base), the network is dense, whereas the density at the top of the nano-network reduces (Fig. 2d). There was also an increase in pore size on the surface of the network with an increase in thickness. The increasing deposition thickness of the nanoparticle-network compresses the pores at the base, resulting in a more densely packed network.

\section{Interaction measurements using Zetasizer and AFM}

Zeta potential is an important physicochemical parameter, as an increase in zeta potential results in a corresponding increase in cell proliferation. ${ }^{25}$ Zeta potentials for the silicon nano-network are measured in relation to deposition thickness, and the results are shown in Figure 3. The graph suggests a linear increase in the negatively charged nanonetwork with an increase in thickness. This can also be correlated to the decreasing particle size. It was earlier shown that as particle size decreases, the zeta potential increases. ${ }^{26}$ A surface with negatively charged zeta potential is hydrophobic and adsorbs proteins onto its surface. This, in turn, leads to higher cell adhesion on the surface with higher zeta potential. ${ }^{27}$ A blank silicon wafer surface was tested as a control. In comparison, the wafers with deposited nano-networks exhibited a considerably higher zeta potential.
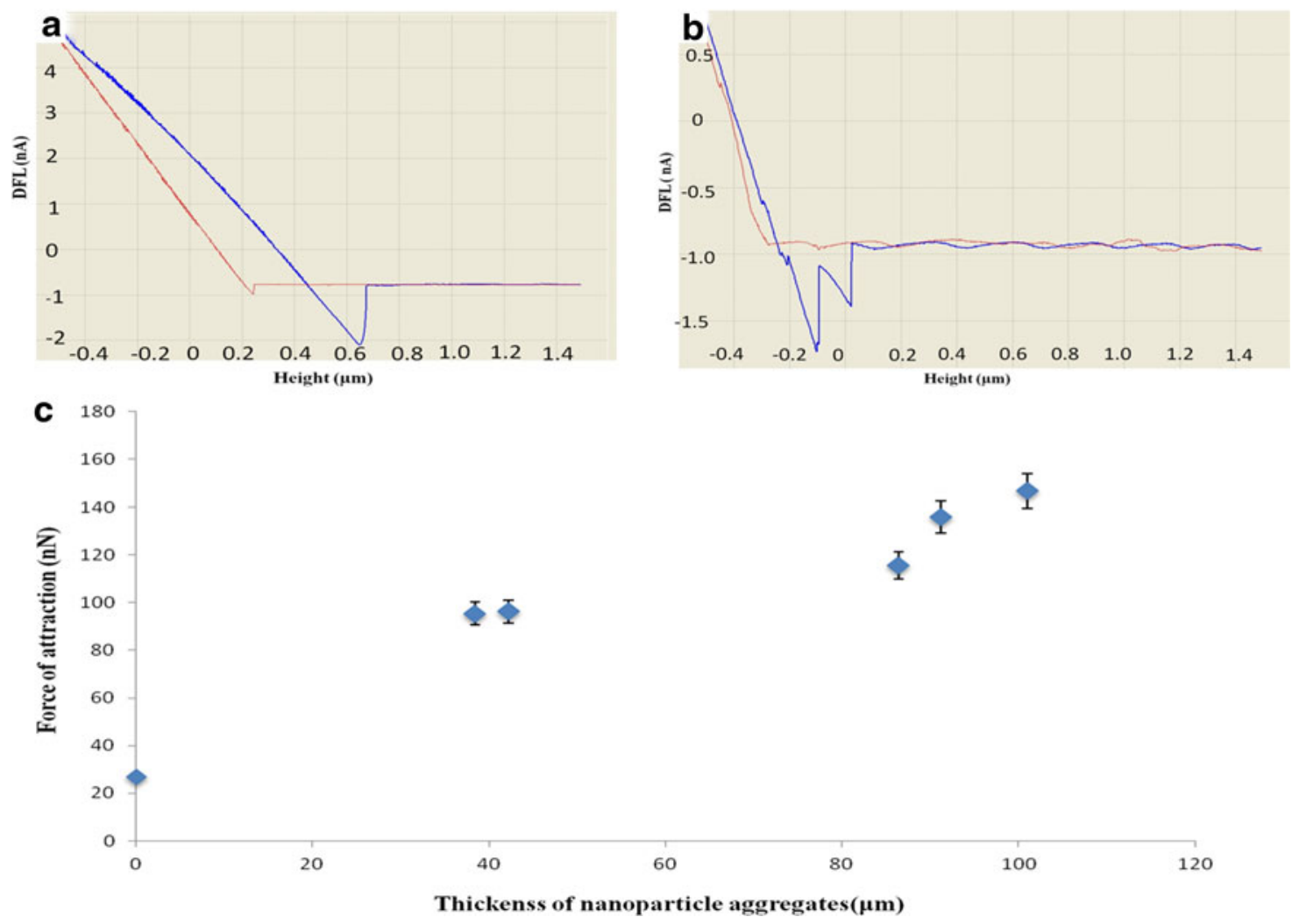

FIG. 4. (a) Force curve from a plain silicon surface (the red lines signify the approach of the tip toward the surface, and the blue lines signify retraction from the surface). (b) Force curve from nano-network deposition. (c) Adhesion forces and deposition thickness. Data at 0 thicknesses refer to the control sample. 
To further study the interaction mechanism, the force of attraction of the nano-network was measured using an AFM, that provides an indication of the interactive forces of the nano-network. The AFM consists of a cantilever that interacts with the substrate. The cantilever is first brought into contact with the sample surface and is then retracted from the surface. It bends downward toward the sample surface due to adhesion forces until a break point is achieved. At this point, the cantilever retracts completely. Figure $4 \mathrm{~b}$ shows an image of the force curve from the surface of silicon deposited with a nano-network. A blank silicon wafer was used as a control sample, and its force curve is depicted in Figure 4a. From the force curve, the adhesion force can be calculated using $F=k \times \Delta l$, where $F$ is the adhesion force $(\mathrm{nN}), k$ is the spring constant $(0.11 \mathrm{~N} / \mathrm{m})$ of the cantilever, and $\Delta l$ is the deflection distance $(\mathrm{nm}){ }^{28}$ The calculated forces are plotted in Figure 4c. The adhesion forces increased dramatically with the deposition of a nanoparticle network. With a thickness of $100 \mu \mathrm{m}$, the adhesion force is seven times higher than that on a plain silicon surface. The initial adherence of cells onto the nano-network depends on their surface characteristics. From the AFM interaction curves, greater forces of attraction were noticed for thicker nano-networks. Therefore, it is expected to see higher cell attachment on thicker substrates.

\section{Bioactivity}

Poor bioactivity will result in fibrous tissue layer formation between the implant and the nearby tissues. This leads to a rejection of the implant from the body. On the other hand, a bioactive material will enhance the formation of hydroxyapatite, promoting cell growth. Hydroxyapatite has a Ca:P ratio of 5:3. It has been shown that a fibrous micro/nano structure is effective in inducing bioactivity. ${ }^{29,30}$ A SBF test was done to study the bioactivity of the nano-network. Bulk silicon showed no apatite formation on its surface for a period of 14 days. Substrates with nano-networks were shown to have apatite formation after just 3 days of incubation. The surface was covered with microspheres of $2-4 \mu \mathrm{m}$ (Fig. 5a). After 10 days, the entire surface was covered by apatite formation. Figure $5 a$ and $b$ shows the formation of hydroxyapatite on silicon nano-networks at both 3 and 10 days, respectively. Figure $5 c$ and d provides the EDX measurement that is used to compare the growth of apatite on silicon surfaces with and without a nano-network. The insets of Figure $5 \mathrm{c}$ and $\mathrm{d}$ indicate the location where measurements were carried out. The EDX analysis showed the presence of sodium, chlorine, and magnesium, in addition to calcium phosphate. The process of formation of calcium phosphate starts with silicon oxides forming $\mathrm{Si}-\mathrm{OH}$ bonds on the surface
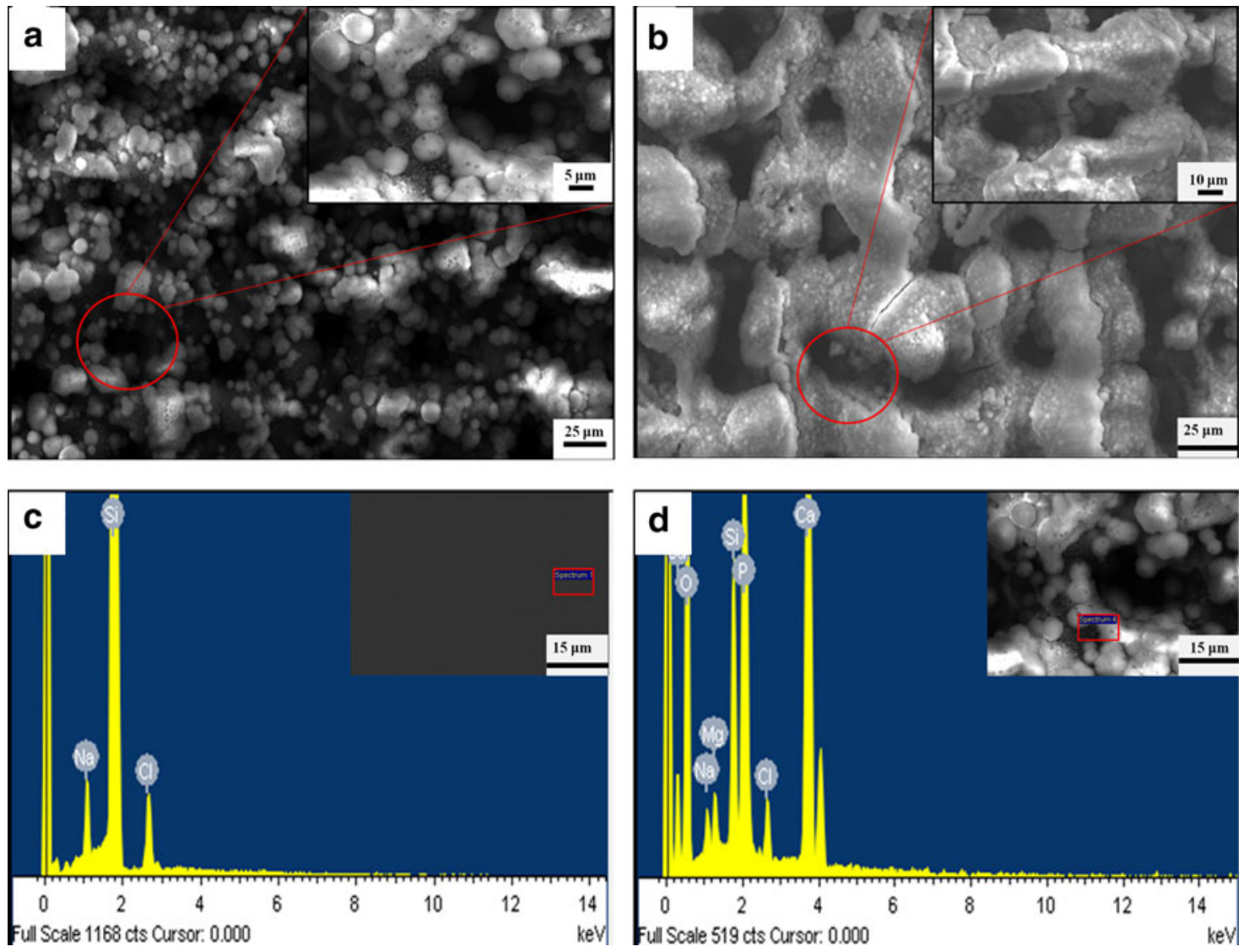

FIG. 5. SEM micrographs of hydroxyapatite formation on silicon nano-network after (a) 3 days and (b) 10 days. Energy-dispersive X-ray spectroscopy showing spectral analysis of silicon samples immersed in simulated body fluid for 3 days: (c) plain silicon; (d) silicon with a nano-network. 
from the water molecules present in the SBF. $\mathrm{Si}-\mathrm{OH}$ has been shown to aid the growth and nucleation of apatite. These molecules attract $\mathrm{Ca}^{2+}$ molecules, forming calcium hydroxide bonds. Finally, these attract phosphate ions forming hydroxyapatites. The structure and physicochemical structure of the nano-networks are responsible for increased bioactivity. Increased bioactivity indicates increased adsorption of proteins, which, in turn, allows for the recruitment of more cells through attachment. ${ }^{31}$

\section{Cell-biomaterial interaction}

An ideal scaffold should aid in cell growth and differentiation apart from eliciting bioactivity. The interaction of $\mathrm{NIH}$ 3T3 mouse embryonic fibroblast cells on a silicon nanonetwork is reported. The relationship between the increasing thickness of the nano-network deposition and cell proliferation was studied, and the results are presented in Figure 6. To study the morphology and attachment of cells, SEM images were taken after $48 \mathrm{~h}$ of incubation (Fig. 7). The SEM images illustrate the healthy proliferation of cells on the substrate. The cells showed multiple filipodia that extended across the nano-network. They formed strong adhesions that are evidenced in large pseudopodial projections. Ruffles on lamellepodia were also noticed. This indicated that cells were spreading well. The cells appear flattened and polarized, which is indicative of migration. The increasing thickness significantly influenced cell proliferation. A $t$-test was performed, and a $p$-value of 0.002 was obtained, showing a significant increase in cell proliferation between control samples and silicon nano-networks. The obtained results reflected that cell proliferation may depend on pore size and/or particle size and also on the ECM-like structure. The fabricated nano-networks consist of nanoscale pores and nanoparticles. $\mathrm{Li}$ et al. noted that even though pore size is in the nano-scale range, during amoeboid movement, cells have the ability to move fibers and manipulate them according to their needs. ${ }^{32}$ Hence, for cells to manipulate fibers, the flexibility of fibers is vital. During SEM and TEM analyses, it was noted that the silicon nano-networks at the top tended to be more flexible with higher porosity and a bigger pore size.

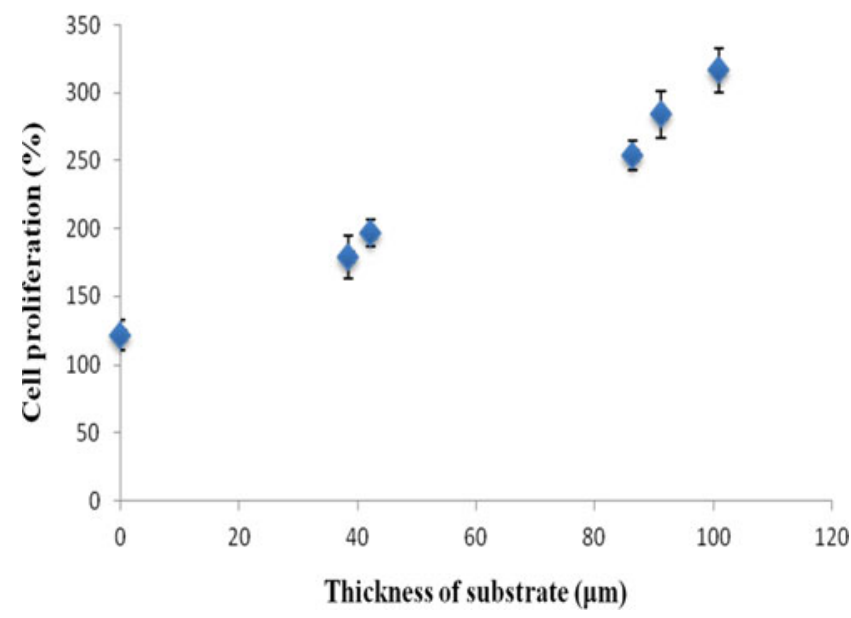

FIG. 6. Increase in proliferation of NIH3T3 fibroblast cells as deposition thickness increases.
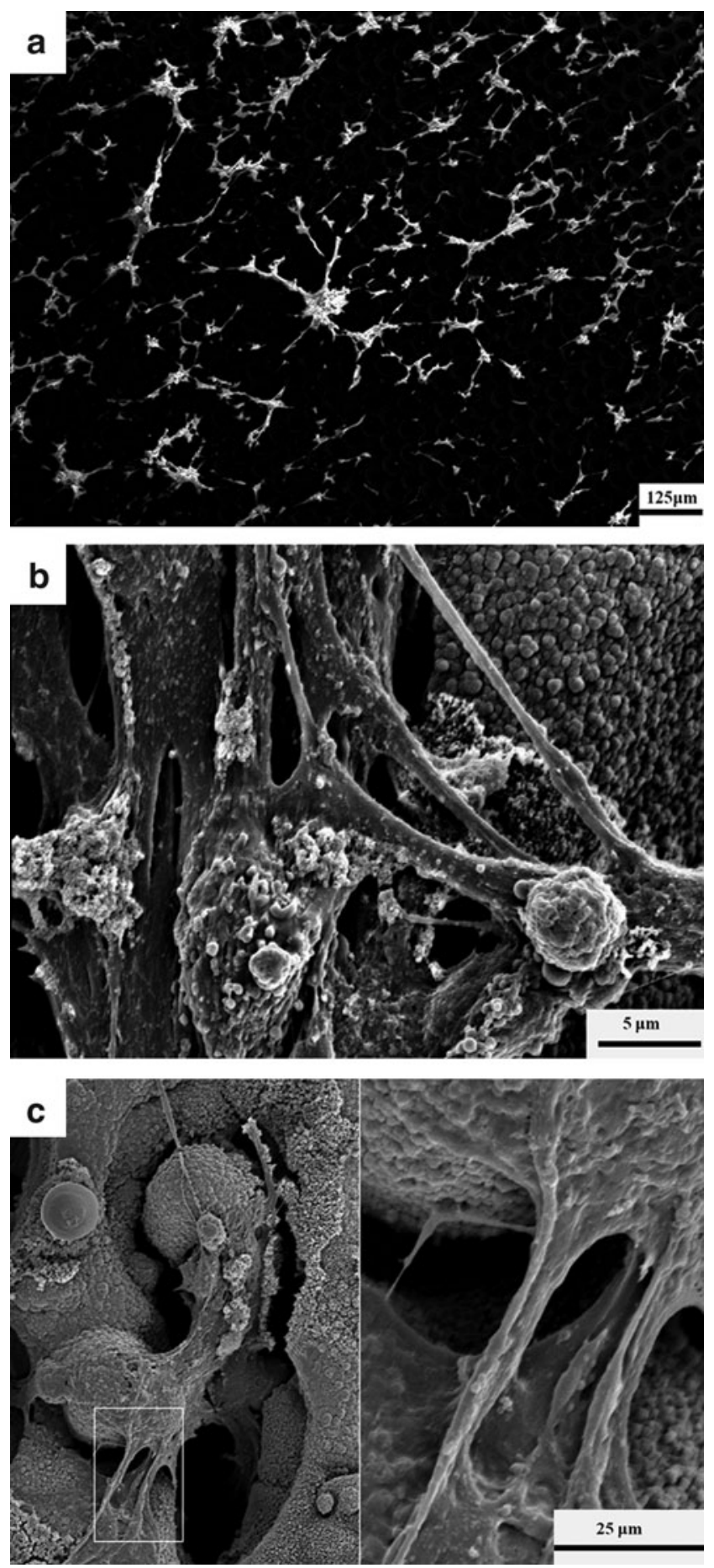

FIG. 7. (a) SEM of fibroblasts on silicon nano-network formed at $10 \mathrm{msec}$. (b, c) Fibroblasts with multiple filopodia spreading out indicate strong adhesion and migration.

This implies that the structure of the nano-network with nanoscale pores plays a direct role in cell proliferation. ${ }^{33}$ Nanoscale features organize the cytoskeleton and membrane receptors such that the signaling between cells is improved. Protein adsorption is also said to improve with these nano features aiding integrin signaling. ${ }^{34}$ Particle size was assumed to elicit differences in proliferation between differing thicknesses of nano-networks. However, further analysis revealed that the 
particles in the nano-networks with different thickness differed only in nano dimensions, and this small change in nano dimensions had no significant effect on cell proliferation. Thus, the cell proliferation studies conducted on the silicon nano-network conclusively proved that nanoscale dimensions have a positive effect on cell growth.

\section{Conclusion}

An ideal scaffold is sought after for its characteristics of biocompatibility, bioactivity, and likeness to ECM. Expensive materials have been well researched at a nano level, but a promising candidate, silicon, was not well studied. In this work, we present a unique way of fabricating a novel interlinked nano-network whose structure and dimensions closely mimic the ECM. This nano-network increased bioactivity, inducing calcium phosphate deposition in 3 days compared with bulk silicon where there was no precipitation after 14 days. Results indicate that the nano structure as well as surface characteristics of this nano-network critically influenced bioactivity. The nanoscale structures presented cells with an ECM-like environment. As a result, we demonstrate a $300 \%$ increase in cell proliferation. Overall, a 1.6-times higher zeta potential and a sevenfold increase in adhesion forces served as contributing factors to the increased cell growth and bioactivity. Nanopores, three dimensionality, and bioactivity not present in previous silicon nanostructures make these nanonetworks suitable replacements for current expensive scaffold biomaterials in the field of tissue engineering.

\section{Acknowledgments}

The authors appreciate the facilities and comments offered by Dr. Anil Kishen (Faculty of Dentistry, University of Toronto) in cell culture experiments. This research was funded by the Natural Sciences and Engineering Research Council of Canada.

\section{Author Disclosure Statement}

The authors report no conflicting financial interests.

\section{References}

1. Badylak SF. The extracellular matrix as a biologic scaffold material. Biomaterials. 2007;28:3587-3593.

2. Smith LA, Ma PX. Nano-fibrous scaffolds for tissue engineering. Colloids Surf B Biointerfaces. 2007;39:125-131.

3. St-Pierre JP, Gauthier M, Lefebvre LP, et al. Three-dimensional growth of differentiating MC3T3-E1 pre-osteoblasts on porous titanium scaffolds. Biomaterials. 2005;26:73197328.

4. Zanello LP, Zhao B, Hu H, et al. Bone cell proliferation on carbon nanotubes. Nano Lett. 2006;6:562-567.

5. Suh H, Park JC, Han DW, et al. A bone replaceable artificial bone substitute: cytotoxicity, cell adhesion, proliferation, and alkaline phosphatase activity. Artif Organs. 2001;25:14-21.

6. Staiger MP, Pietak AM, Huadmai J, et al. Magnesium and its alloys as orthopedic biomaterials: a review. Biomaterials. 2006;27:1728-1734.

7. Ducheyne P, Qiu Q. Bioactive ceramics: the effect of surface reactivity on bone formation and bone cell function. Biomaterials 1999;20:2287-2303.

8. Coffer JL, Whitehead MA, Nagesha DK, et al. Porous siliconbased scaffolds for tissue engineering and other biomedical applications. Phys Status Solidi A Appl Res 2005;202: 1451-1455.

9. Brovarone CV, Verne E, Robiglio L, et al. Development of glass-ceramic scaffolds for bone tissue engineering: characterization, proliferation of human osteoblasts and nodule formation. Acta Biomater. 2007;3:199-208.

10. Canham LT. Bioactive silicon structure fabrication through nanoetching techniques. Adv Mater. 1995;7:1033-1037.

11. Sun W, Puzas JE, Sheu TJ, et al. Porous silicon as a cell interface for bone tissue engineering. Phys Status Solidi A Appl Res. 2007;204:1429-1433.

12. Agrawal AA, Nehilla BJ, Reisig KV, et al. Porous nanocrystalline silicon membranes as highly permeable and molecularly thin substrates for cell culture. Biomaterials. 2010;31:54085417.

13. Ranella A, Barberoglou M, Bakogianni S, et al. Tuning cell adhesion by controlling the roughness and wettability of 3D micro/nano silicon structures. Acta Biomater. 2010;6: 2711-2720.

14. Lee J, Chu BH, Chen $\mathrm{KH}$, et al. Randomly oriented, upright $\mathrm{SiO} 2$ coated nanorods for reduced adhesion of mammalian cells. Biomaterials. 2009;30:4488-4493.

15. Thian ES, Huang J, Best SM, et al. The response of osteoblasts to nanocrystalline silicon-substituted hydroxyapatite thin films. Biomaterials. 2006;27:2692-2698.

16. Kim W, Ng JK, Kunitake ME, et al. Interfacing silicon nanowires with mammalian cells. J Am Chem Soc. 2007;129:72287229 .

17. Nazarov R, Jin HJ, Kaplan DL. Porous 3-D scaffolds from regenerated silk fibroin. Biomacromolecules. 2004;5:718-726.

18. Behan BL, DeWitt DG, Bogdanowicz DR, et al. Single-walled carbon nanotubes alter Schwann cell behavior differentially within 2D and 3D environments. J Biomed Mater Res A. 2010;96:46-57.

19. Cukierman E, Pankov R, Yamada KM. Cell interactions with three-dimensional matrices. Curr Opin Cell Biol. 2002;14: 633-639.

20. Tan B, Venkatakrishnan K. Synthesis of fibrous nanoparticle aggregates by femtosecond laser ablation in air. Opt Express. 2009;17:1064-1069.

21. Kokubo T, Takadama H. How useful is SBF in predicting in vivo bone bioactivity. Biomaterials. 2006;27:2907-2915.

22. Mosmann T. Rapid colorimetric assay for cellular growth and survival: application to proliferation and cytotoxicity assays. J Immunol Methods. 1983;65:55-63.

23. Tillack MS, Blair DW, Harilal SS. The effect of ionization on cluster formation in laser ablation plumes. Nanotechnology. 2004;15:390-403.

24. Tavangar A, Tan B, Venkatakrishnan K. Single-step fabrication of microfluidic channels filled with nanofibrous membrane using femtosecond laser irradiation. J Micromech Microeng. 2010;20:085016; DOI: 10.1088/0960-1317/20/8/ 085016.

25. Kishida A, Iwata H, Tamada Y, et al. Cell behaviour on polymer surfaces grafted with non-ionic and ionic monomers. Biomaterials. 1991;12:786-792.

26. Sonavane G, Tomoda K, Makino K. Biodistribution of colloidal gold nanoparticles after intravenous administration: effect of particle size. Colloids Surf Biointerfaces. 2008;66:274-280.

27. Wachem PB, Hogt AH, Beugeling T, et al. Adhesion of cultured human endothelial cells onto methacrylate polymers with varying surface wettability and charge. Biomaterials. 1987;8: 323-328. 
28. Fang HH, Chan KY, Xu LC. Quantification of bacterial adhesion forces using atomic force microscopy (AFM). J Microbiol Methods. 2000;40:89-97.

29. Fujibayashi S, Neo M, Kim HM, et al. Osteoinduction of porous bioactive titanium metal. Biomaterials. 2004;25:443-450.

30. Tavangar A, Tan B, Venkatakrishnan K. Synthesis of biofunctionalized three dimensional titania nanofibrous structures using femtosecond laser ablation. Acta Biomater. 2011;7:2726-2732.

31. Pietak AM, Reid JW, Stott MJ, et al. Silicon substitution in the calcium phosphate bioceramics. Biomaterials. 2007;28:40234032.

32. Li WJ, Laurencin CT, Caterson EJ, et al. Electrospun nanofibrous structure: a novel scaffold for tissue engineering. J Biomed Mater Res. 2012;60:613-621.
33. Choi $\mathrm{CH}$, Hagvall $\mathrm{SH}, \mathrm{Wu} \mathrm{BM}$, et al. Cell interaction with three-dimensional sharp-tip nanotopography. Biomaterials. 2007;28:1672-1679.

34. Stevens MM, George JH. Exploring and engineering the cell surface interface. Science. 2005;310:1135-1138.

Address correspondence to: Bo Tan, PhD Department of Aerospace Engineering Ryerson University 350 Victoria Street Toronto M5B 2K3, ON Canada

E-mail: tanbo@ryerson.ca 